\title{
An online framework for introducing STI point-of-care tests in Pacific island countries and areas
}

\author{
Lisa Natolia and Benjamin Coghlan ${ }^{a}$ \\ Correspondences to Benjamin Coghlan (e-mail: coghlan@burnet.edu.au).
}

S exually transmitted infections (STIs) are an important public health issue in Pacific island countries and areas. Diagnosis is challenging, often requiring laboratory facilities and technical expertise rarely available. Patients seldom have results before they leave the health facility, with management primarily based on symptoms. As the delay between testing and treatment increases, so does the potential for complications of infection for the individual, the likelihood of STI transmission to other sexual partners and the chance the client will not return for follow-up. Effective tests that can be used at the point of care (POC) can conceivably overcome these consequences of delayed diagnosis. Such tests for STIs are becoming more available and affordable, with the potential to improve STI control. ${ }^{1}$ However, the introduction of $\mathrm{POC}$ tests is complex, ${ }^{2}$ requiring consideration of a range of implications to operationalize testing successfully and minimize potential harms.

The Burnet Institute was funded by the Secretariat of the Pacific Community to support ministries of health in the region to explore ways to integrate POC testing into existing STI management and control strategies. A participatory consultation process was undertaken with members of the Pacific Regional STI Working Group, selected ministry of health representatives and regional experts to develop an online 'toolkit' to provide a framework for health managers to critically appraise the suitability of POC testing for STIs in their country, to ensure a smooth introduction of the test if deemed appropriate, and to minimize harms. While there are several resources that describe these aspects in detail, ${ }^{3-5}$ they are often focused on a specific infection with formatting that precludes easy use by health managers in the Pacific.
The web-based framework consists of three sections that can be read as sequential steps or referred to individually as needed. This structure means that those seeking to review, strengthen or change their existing approach to STI testing may also find the toolkit useful. Section 1, Initial Assessment, provides advice on how to weigh the benefits and costs of introducing an STI POC test. It also includes background information on key definitions and technical concepts. Section 2, Piloting the STI POC Test, outlines the steps in preparing for and implementing a pilot test to determine how well the POC test performs in the local setting. This is an important stage before wider introduction of a POC test. Section 3, Scaling Up, briefly discusses the principles of using the POC test on a wider scale. The toolkit also includes downloadable tools to help health managers with particular steps and links to relevant printed and organizational resources. The challenges and complexities of introducing STI POC tests are highlighted with snapshots of lessons learnt from the region.

The online toolkit will be road-tested later this year to ensure that language, content and usability match the needs of users in the Pacific. The toolkit will be revised following the trial, and hosted on the Burnet Institute web site. Those wishing to have an advanced look at the toolkit can contact the authors at the e-mail address listed above; feedback is welcome.

\section{References:}

1. Ward P. Near-patient testing will improve the control of sexually transmitted infections: the arguments in favour. Sexually Transmitted Infections, 2006, 82:506-508. doi:10.1136/ sti.2005.017962 pmid:17151037

2. Peeling RW et al. Rapid tests for sexually transmitted infections (STIs): the way forward. Sexually Transmitted Infections,

Macfarlane Burnet Institute for Medical Research and Public Health, Centre for International Health, Melbourne, Australia. Submitted: 17 August 2012; Published: 11 September 2012

doi: 10.5365/wpsar.2012.3.3.005 
2006, 82 (Suppl 5):v1-6. doi:10.1136/sti.2006.024265 pmid: 17151023

3. Rapid Syphilis Test Toolkit: A Guide to Planning, Management and Implementation. London School of Hygiene and Tropical Medicine, $2011 \quad$ (http://www.Ishtm.ac.uk/itd/crd/research/ rapidsyphilistoolkit/, accessed 30 July 2012).

4. Banoo S et al.; TDR Diagnostics Evaluation Expert Panel. Evaluation of diagnostic tests for infectious diseases: general principles. Nature Reviews Microbiology, 2010, 8 Suppl:S17-29. pmid:21548184

5. UNAIDS/WHO Working Group on Global HIV/AIDS/STI Surveillance. Guidelines for Using HIV Testing Technologies in Surveillance: Selection, Evaluation, and Implementation. Geneva, World Health Organization and Joint United Nations Programme on HIV/ AIDS, 2001 (http://www.who.int/hiv/pub/surveillance/en/guideline sforUsingHIVTestingTechs_E.pdf, accessed 30 July 2012). 